\title{
SCHAUDER'S FIXED-POINT THEOREM IN APPROXIMATE CONTROLLABILITY PROBLEMS
}

\author{
ARTUR BABIARZ $^{a, *}$, JERZY KLAMKA $^{a}$, MiCHAŁ NIEZABITOWSKI $^{a}$ \\ ${ }^{a}$ Institute of Automatic Control \\ Silesian University of Technology, Akademicka 16, 44-100 Gliwice, Poland \\ e-mail: \{artur.babiarz, jerzy.klamka, michal.niezabitowski\}@polsl.pl
}

\begin{abstract}
The main objective of this article is to present the state of the art concerning approximate controllability of dynamic systems in infinite-dimensional spaces. The presented investigation focuses on obtaining sufficient conditions for approximate controllability of various types of dynamic systems using Schauder's fixed-point theorem. We describe the results of approximate controllability for nonlinear impulsive neutral fuzzy stochastic differential equations with nonlocal conditions, impulsive neutral functional evolution integro-differential systems, stochastic impulsive systems with control-dependent coefficients, nonlinear impulsive differential systems, and evolution systems with nonlocal conditions and semilinear evolution equation.
\end{abstract}

Keywords: approximate controllability, Banach space, Hilbert space, Schauder's fixed-point theorem, infinite-dimensional space.

\section{Introduction}

Controllability is one of the most important notions in modern mathematical control theory (see the works of Bashirov and Kerimov (1997), Bashirov and Mahmudov (1999), Benchohra and Ouahab (2005), Gorniewicz et al. (2005) and the references therein). Using the assumption that the system considered is controllable, we are able to solve many problems of control theory such as stabilizability, optimal control and pole assignment. The notion of controllability means that it is possible to steer a dynamic system from an arbitrary initial state to an arbitrary final state using a set of admissible controls. There exist many criteria and definitions of controllability. They depend both on the constraints on the control signal and the state equation. It should be noticed that in infinite-dimensional spaces there exist linear subspaces which are not closed. We can distinguish two concepts of controllability in the case of infinite-dimensional systems. Exact controllability means that the system can be steered to an arbitrary final state. Approximate controllability enables us to steer the system to an arbitrary small neighborhood of the final state. It is self-evident that approximate controllability is essentially a weaker notion

*Corresponding author than exact controllability. As a result, the latter always implies the former, but the converse statement is not true in general. In the finite-dimensional case, the notions of approximate and exact controllability coincide. Sikora and Klamka (2012) focused on the controllability problem of linear stochastic systems. The research presented by Czornik and Świerniak (2001; 2004; 2005) considered controllability of jump systems. Moreover, controllability of switched linear systems was investigated by Babiarz et al. (2015a; 2015b; 2016).

In the work of Klamka (2000) constrained approximate controllability problems for linear abstract dynamic systems with a linear unbounded control operator and piecewise polynomial controls were investigated. Approximate controllability results for first order linear and nonlinear systems can be found in the work of Henríquez (2008). The research fundamental of the controllability problem of nonlinear systems is the fixed-point theorem (Gorniewicz et al., 1991; Kryszewski and Zezza, 1994; Bader and Kryszewski, 1994; Bader et al., 1996; Przeradzki, 2012).

Some problems of controllability of infinite-dimensional nonlinear systems were studied by Zhou (1983), Naito (1989), Bian (1999), as well as Balachandran and Dauer (2002). Schauder's fixed 
point theorem was used by Dauer and Mahmudov (2002) in relation to approximate controllability of first order functional differential equations with finite delay. Approximate controllability of backward stochastic evolution equations in Hilbert space is considered by Dauer et al. (2006).

The notions of approximate controllability of nonlinear impulsive differential systems and stochastic systems with unbounded delay were investigated by Sakthivel et al. (2007; 2010). The work of Zang and $\mathrm{Li}$ (2013) is devoted to the concept of approximate controllability of fractional impulsive neutral stochastic differential equations with nonlocal conditions. Controllability considerations of stochastic systems can be found in the works of Arapostathis et al. (2001), Mahmudov (2001a; 2001b) or Mahmudov and Zorlu (2003). Controllability of impulsive differential inclusions by using a fixed point theorem was discussed by Benchohra et al. (2004). Schaefer's fixed point theorem was used to establish sufficient conditions for controllability of impulsive functional differential systems (Li et al., 2006). Controllability of impulsive functional systems with infinite delay were investigated by Chang (2007). Approximate controllability for various classes of evolution stochastic differential equations was studied by Dubov and Mordukhovich (1978) as well as Sirbu and Tessitore (2001).

Jeong and Roh (2006) studied approximate controllability for a semilinear retarded control system. A discussion of approximate controllability and approximate null controllability of control systems governed by a class of abstract semilinear integro-differential equations can be found in the work of Wang (2006). Approximate controllability for semilinear control systems is discussed by Do (1989), George (1995) and Mahmudov (2003). Controllability of semilinear integrodifferential systems in Banach spaces was investigated by Lasiecka and Triggiani (1991) as well as Balachandran and Sakthivel (2001).

\section{Background}

2.1. Fundamental notation. Let us introduce the following necessary notation:

- H, $K$ and $U$ are Hilbert spaces and $K, U$ are separable;

- $\mathcal{L}(K, H)$ is the space of all bounded operators from $K$ to $H$;

- $\psi^{*} \in \mathcal{L}(H, K)$ is the adjoint operator for $\psi \in$ $\mathcal{L}(K, H)$;

- $(e(n))_{n \in \mathbb{N}}$ is a complete orthonormal basis in $K$;
- $A$ is a closed densely defined operator generating an analytic semigroup $\{S(t) ; t>0\}$ on $H$ with inner product $\langle\cdot, \cdot\rangle$ and norm $\|\cdot\|$;

- $A^{\alpha}: H_{\alpha} \subset H \rightarrow H$ is a fractional power operator with domain $H_{\alpha}$;

- in $H_{\alpha}$ we define the norm, $\|x\|_{\alpha}:=\left\|A^{\alpha} x\right\|$ for $x \in$ $H_{\alpha}$ (with this norm $H_{\alpha}$ is a Banach space (cf. Pazy, 2012);

$-\mathcal{C}_{\alpha}=\mathcal{C}\left([-r, 0], H_{\alpha}\right)$ is the space of all continuous functions from $[-r, 0]$ into $H_{\alpha}, 0<r<\infty$;

- $B$ is a bounded linear operator from $U$ into $H$;

- $(\Omega, \mathcal{F}, P)$ is a probability space with a probability measure $P$ on $\Omega$;

- E signifies the expected value;

- $L_{p}(\Omega, H)$ is the space of all functions $V: \Omega \rightarrow H$ such that $\mathbf{E}\|V\|^{p}<\infty$;

$-\left\{\mathcal{F}_{t}: t \geq 0\right\}$ is an increasing and right continuous family of complete sub- $\sigma$-algebras of $\mathcal{F}$;

- $\phi$ is an $\mathcal{F}_{0}$-measurable stochastic process;

- if $T>0, \mathcal{X}$-metric space and $F: \Omega \rightarrow \mathcal{X}$, then $F$ is called $\mathcal{F}_{t}$-adapted if $F$ is $\mathcal{F}_{t}$-measurable for almost all $t \in[0, T]$, and is called $\mathcal{F}_{0}$-adapted if it is $\mathcal{F}_{0}$-measurable for almost all $t \in[-r, 0]$;

- $X(t): \Omega \rightarrow H_{\alpha}, t \geq-r$, stands for a continuous $\mathcal{F}_{t}$-adapted, $H_{\alpha}$-valued stochastic process;

$-X_{t}: \Omega \rightarrow \mathcal{C}_{\alpha}, t \leq 0$ is defined by

$$
X_{t}(\omega)=\{X(t+s)(\omega): s \in[-r, 0]\}
$$

and it is called a $\mathcal{C}_{\alpha}$-valued stochastic process;

$-\left(\beta_{n}(t)\right)_{n \in \mathbb{N}}$ is the sequence of real-valued one-dimensional standard Brownian motions mutually independent over $(\Omega, \mathcal{F}, P)$;

- for a sequence $\left(\lambda_{n}\right)_{n \in \mathbb{N}}, \lambda_{n} \geq 0$, we define

$$
W(t)=\sum_{n=1}^{\infty} \sqrt{\lambda_{n}} \beta_{n}(t) e_{n}, \quad t \geq 0
$$

then $W(t)$ is a $K$-valued Wiener process with a finite trace nuclear covariance operator $Q \geq 0$, where $Q \in$ $\mathcal{L}(K, K)$ is the operator with the property $Q e_{n}=$ $\lambda_{n} e_{n}$ and a finite trace

$$
\operatorname{tr} Q=\sum_{n=1}^{\infty} \lambda_{n}<\infty
$$


- $\mathcal{F}_{t}=\sigma(W(s): 0 \leq s \leq t)$ signifies the $\sigma$-algebra generated by $W$ and $\mathcal{F}_{t}=\mathcal{F}$;

- let $\psi \in \mathcal{L}(K, H)$ and define

$$
\|\psi\|_{Q}^{2}=\operatorname{tr}\left[\psi Q \psi^{*}\right]=\sum_{n=1}^{\infty}\left\|\sqrt{\lambda_{n}} \psi e_{n}\right\|^{2} ;
$$

- $\psi$ is a $Q$-Hilbert-Schmidt operator if $\|\psi\|_{Q}<\infty$;

- $L_{2}^{0}(K, H)$ is the space of all $Q$-Hilbert-Schmidt operators from $K$ into $H$;

- here and subsequently,

$$
\begin{gathered}
f:[0, \infty) \times \mathcal{C}_{\alpha} \times H \rightarrow H, \\
\sigma:[0, \infty) \times \mathcal{C}_{\alpha} \times H \rightarrow L_{2}^{0}(K, H)
\end{gathered}
$$

and

$$
g:[0, \infty) \times[0, \infty) \times \mathcal{C}_{\alpha} \rightarrow H
$$

are measurable mappings such that $f(t, 0,0)$, $\sigma(t, 0,0)$ and $g(t, s, 0)$ are locally bounded in $H$-norm, $L_{2}^{0}(K, H)$-norm and $H$-norm, respectively;

- $M \mathcal{C}_{\alpha}(0, p), p>2$, is the space of all $\mathcal{F}_{0}$-measurable $\mathcal{C}_{\alpha}$-valued functions $\varsigma: \Omega \rightarrow \mathcal{C}_{\alpha}$ with the norm

$$
\mathbf{E}\|\varsigma\|_{\mathcal{C}_{\alpha}}^{p}=\mathbf{E}\left\{\sup _{-r \leq s \leq 0}\left\|A^{\alpha} \varsigma(s)\right\|^{p}\right\}<\infty ;
$$

- $L_{p}^{\mathcal{F}}([0, T], H)$ is the closed subspace of

$$
L_{p}([0, T] \times \Omega \times \Omega, H)
$$

consisting of all $\mathcal{F}_{t}$-adapted processes;

$-\mathcal{C}\left([-r, T], L_{p}(\Omega, H)\right)$ is the Banach space of all continuous maps from $[-r, T]$ into $L_{p}(\Omega, H)$ satisfying the condition

$$
\sup _{t \in[-r, T]} \mathbf{E}\|X(t)\|^{p}<\infty
$$

- $\mathcal{H}_{p}$ is the closed subspace of all continuous processes $X$ with trajectories in $\mathcal{C}\left([-r, T], L_{p}(\Omega, H)\right)$ with

$$
\begin{aligned}
& \|X\|_{\mathcal{H}_{p}} \\
& =\left(\sup _{t \in[0, T]} \mathbf{E}\left\|X_{t}\right\|_{\mathcal{C}}^{p}\right)^{\frac{1}{p}} \\
& =\left(\sup _{t \in[0, T]} \mathbf{E} \sup _{-r \leq s \leq 0}\left\|X_{t}(\omega)\right\|_{\mathcal{C}}^{p}\right)^{\frac{1}{p}}<\infty ;
\end{aligned}
$$

- $\mathcal{P C}((\infty, b], X)$ is a Banach space with the norm

$$
\|x\|_{\mathcal{P C}}=\sup _{t \in[0, b]}\|x(t)\|
$$

and

$$
\begin{aligned}
\mathcal{P C} & ((-\infty, b], X) \\
= & \{x: x \text { is function from }(-\infty, b] \text { into } X \text { such that } \\
& x(t) \text { is continuous at } t \neq t_{k} \text { and left continuous } \\
& \text { at } t=t_{k} \text { and the right limit } x\left(t_{k}^{+}\right) \text {exists } \\
& \text { for } k=1,2, \ldots, m\} .
\end{aligned}
$$

$-\mathcal{B}_{h}$ is the abstract phase space defined in Section 3.2

2.2. Schauder's fixed-point theorem. Below we present Schauder's fixed-point theorem.

Theorem 1. (Schauder's theorem) (Kulmin, 2004). Every continuous operator that maps a closed convex subset of a Banach space into a compact subset of itself has at least one fixed point.

Theorem 2. (Kulmin, 2004). Let $\mathcal{X}$ be a locally convex topological vector space, and let $\mathcal{K} \subset \mathcal{X}$ be a nonempty, compact, and convex set. Then, given any continuous mapping $f: \mathcal{K} \rightarrow \mathcal{K}$, there exists $x \in \mathcal{K}$ such that $f(x)=x$.

\section{Approximate controllability of semilinear systems}

This section presents results concerning approximate controllability of various kinds of dynamic systems described by semilinear state equations.

3.1. Nonlinear impulsive neutral fuzzy stochastic differential equations with nonlocal conditions. Narayanamoorthy and Sowmiya (2015) consider the approximate controllability for nonlinear impulsive neutral fuzzy stochastic differential equations with nonlocal conditions given by

$$
\begin{gathered}
\mathrm{d}[x(t)-h(t, x(t))] \\
=A[x(t)-h(t, x(t))] \mathrm{d} t+B u(t) \mathrm{d} t \\
+f(t, x(t)) \mathrm{d} t+g(t, x(t)) \mathrm{d} W(t), \\
\Delta x\left(t_{i}\right)=x\left(t_{i}^{+}\right)-x\left(t_{i}^{-}\right), \quad i=1,2, \ldots, m, \\
\quad x(0)+\mu(x)=x_{0}, \quad x \in X, \\
\quad t \in J=[0, a], \quad t \neq t_{i},
\end{gathered}
$$

where

- the state variable $x(\cdot)$ takes values in a real separable Hilbert space $X$ with an inner product $(\cdot, \cdot)$ and the induced norm $\|\cdot\|$; 
- the control function $u(\cdot)$ takes values in $L^{2}(J, U)$, a Banach space of admissible control functions for a separable Hilbert space $U$ and $J=[0, b], \Omega=$ $\{(t, s): 0 \leq s \leq t \leq b\}$

- $A=A(t, x)$ constitutes the infinitesimal generator of a $C_{0}$-semigroup in $X$;

- $B$ is a bounded linear operator from $U$ into $X$;

- $f$ and $g$ are continuous and compact functions, and $f: J \rightarrow X$ and $g: \Omega \rightarrow X$ are measurable mappings in $X$-norm

- a neutral variable $h$ is continuous and compact;

- $h: X \rightarrow X$ is a measurable mapping;

- the function $\mu: P C(J, X) \rightarrow X$ is continuous;

- the impulsive function $I_{i}: X \rightarrow X$ is compact;

- the fixed time $t_{i}$ satisfies $0=t_{0}<t_{1}<t_{2}<\ldots<$ $t_{m}<a$;

- $x\left(t_{i}^{+}\right)$and $x\left(t_{i}^{-}\right)$denote respectively the right and left limits of $x(t)$ at $t=t_{i}$;

- $\Delta x\left(t_{i}\right)=x\left(t_{i}^{+}\right)-x\left(t_{i}^{-}\right)$represents the jump in the state $X$ at time $t_{i}$;

- $I_{i}$ determines the size of the jump.

Here, we introduce some essential definitions.

Definition 1. (Narayanamoorthy and Sowmiya, 2015) $A(t): 0 \leq t \leq b$ generates a unique linear evolution system $U(t, s): 0 \leq s \leq t \leq b$ satisfying the following properties:

1. $U(t, s) U(s, \tau)=U(t, \tau)$ and $U(t, t) x=x$ for every $s \leq \tau \leq t$ and all $x \in X$;

2 . for every $x \in X$, the function for $(t, s) \rightarrow U(t, s) x$ is continuous and $U(t, s) \in L(X)$ for every $t \geq s$;

3. for $0 \leq s \leq t \leq b$, the function $t \rightarrow U(t, s),(s, t] \in$ $L(X)$ is differentiable with

$$
\frac{\partial U(t, s)}{\partial t}=A(t) U(t, s)
$$

Definition 2. (Narayanamoorthy and Sowmiya, 2015) A stochastic process $x$ is said to be a mild solution of (1) if the following conditions are satisfied:

1. $X(t, \omega)$ is a measurable function from $J \times \Omega$ to $X$ and $x(t)$ is $F_{t}$-adapted;

2. $E\|x(t)\|^{2}<\infty$ for all $t \in J$;
3. $\Delta x\left(\tau_{i}\right)=x\left(\tau_{i}^{+}\right)-x\left(\tau_{i}^{-}\right)=I_{i}\left(x\left(\tau_{i}\right)\right), x \in X$ and $1 \leq i \leq m$;

4. for each $u \in L_{2}^{F}(J, U)$, the process $x$ satisfies the following integral equation:

$$
\begin{aligned}
x(t)= & U(t, 0)\left[x^{\prime}-\mu(x)\right] \\
& +\int_{0}^{t} U(t, s) B u(s) \mathrm{d} s \\
& +\int_{0}^{t} U(t, s) f(s, x(s)) \mathrm{d} s \\
& +\int_{0}^{t} U(t, s) g(s, x(s)) \mathrm{d} W(s) \\
& +\sum_{0 \leq t_{i} \leq t} U\left(t, t_{i}\right) I\left(x\left(t_{i}^{-}\right)\right), \quad t \in J .
\end{aligned}
$$

Definition 3. (Narayanamoorthy and Sowmiya, 2015) Let $x_{b}\left(x_{0} ; u\right)$ be the state value of the system (1) at a terminal time $b$ corresponding to the control $u$ and the initial value $x_{0}=\phi \in \mathcal{B}_{h}$. Then the set $\mathcal{R}\left(b, x_{0}\right)=$ $\left\{x_{b}\left(x_{0} ; u\right)(0): u(\cdot) \in L^{2}(J, U)\right\}$ is called the reachable set at the terminal time $b$ and its closure in $X$ is denoted by $\overline{\mathcal{R}\left(b, x_{0}\right)}$.

Definition 4. (Narayanamoorthy and Sowmiya, 2015) If $\overline{\mathcal{R}\left(b, x_{0}\right)}=X$, then the system (1) is approximately controllable on the interval $J$.

It is convenient at this point to define operators

$$
\begin{gathered}
\Gamma_{0}^{a}=\int_{0}^{a} U(a, s) B B^{*} U^{*}(a, s) \mathrm{d} s, \\
\mathcal{R}\left(\alpha, \Gamma_{0}^{a}\right)=\left(\alpha I+\Gamma_{0}^{a}\right)^{-1} .
\end{gathered}
$$

In order to present results of approximate controllability for nonlinear impulsive neutral fuzzy stochastic differential equations under nonlocal conditions by using Schauder's fixed point theorem, the following assumptions should be introduced (Narayanamoorthy and Sowmiya, 2015)

Assumption 1. $\alpha R\left(\alpha, \Gamma_{0}^{a}\right) \rightarrow 0$ as $\alpha \rightarrow 0^{+}$in the strong operator topology.

Assumption 2. When $t>s>0$, the strongly continuous semigroup of bounded linear operators $U(t, s)$ generated by $A(t)$ is compact and there exist constants $M_{1}>0$, $M_{2}>0$ such that

$$
\left\|[U(t, s)]^{\alpha}\right\| \leq M_{1}^{\alpha}, \quad\left\|[U(t, s)]^{\alpha}\right\| \leq M_{2}^{\alpha} .
$$

Assumption 3. The nonlinear function $g: J \times B_{h} \times X \rightarrow$ $X$ is continuous, strongly measurable and there exists $L_{g}>0$ such that

$\left\|[g(t, \phi, x)]^{\alpha}\right\| \leq L_{g}^{\alpha} \quad$ for all $(t, \phi, x) \in J \times B_{h} \times X$, where $B_{h}$ is a Banach space. 
Assumption 4. $I_{k} \in C(X, X)$ and there exists a constant $d_{k}^{\alpha}$ such that

$$
\left|\left[I_{k}(x)\right]^{\alpha}\right| \leq d_{k}^{\alpha}(|x|), \quad k=1,2, \ldots, m,
$$

for each $x \in X$, and

$$
\liminf \frac{d_{k}(\rho)}{\rho}=\lambda_{k} \leq \infty, \quad k=1,2, \ldots, m .
$$

Theorem 3. (Narayanamoorthy and Sowmiya, 2015). If Assumptions 14 are satisfied, then the system (1) is approximately controllable on $\mathrm{J}$.

3.2. Impulsive neutral functional evolution integrodifferential system. A special case of the semilinear system is an impulsive neutral functional evolution integro-differential system given by (Radhakrishnan and Balachandran, 2011)

$$
\begin{aligned}
& \frac{\mathrm{d}}{\mathrm{d} t}\left[x(t)+g\left(t, x_{t}\right)\right] \\
& =A(t) x(t)+\int_{0}^{t} G(t, s) x(s) \mathrm{d} s+(B u)(t) \\
& \quad+f\left(t, x_{t}, \int_{0}^{t} h\left(t, s, x_{s}\right) \mathrm{d} s\right), \\
& \quad t \in J, \quad t \neq t_{k}, \quad k=1,2, \ldots, m, \\
& I_{k}\left(x\left(t_{k}^{-}\right)\right)=x\left(t_{k}^{+}\right)-x\left(t_{k}^{-}\right), \quad x_{0}=\phi \in \mathcal{B}_{h},
\end{aligned}
$$

where $\mathcal{B}_{h}$ is the abstract phase space defined as follows:

$$
\begin{array}{r}
\mathcal{B}_{h}=\{\psi:(-\infty, 0] \rightarrow X, \text { such that for any } c>0, \\
\left.\left.\psi\right|_{[-c, 0]} \in \mathcal{B} \text { and } \int_{\infty}^{0} h(s)\|\psi\|_{[c, 0]} \mathrm{d} s<\infty\right\}
\end{array}
$$

and for any $b>0$, we can define

$$
\begin{gathered}
\mathcal{B}=\{\psi:[-b, 0] \rightarrow X \text { such that } \psi(t) \\
\text { is bounded and measurable }\}
\end{gathered}
$$

and equip the space $\mathcal{B}$ with the norm

$$
\|\psi\|_{[-b, 0]}=\sup _{s \in[-b, 0]}|\psi(s)| \quad \text { for all } \psi \in \mathcal{B} .
$$

Moreover,

- the state $x(\cdot)$ takes values in the Hilbert space $X$ with a norm $\|\cdot\|$;

- $x_{t}$ represents the function $x_{t}:(-\infty, 0] \rightarrow X$ defined by $x_{t}(\theta)=x(t+\theta), \infty<\theta<0$, which belongs to $\mathcal{B}_{h}$;

- the control $u(\cdot)$ is given in $L^{2}(J, V)$, a Hilbert space of admissible control functions with $V$ as a Hilbert space and thereby $J=[0, b]$;
$-D=\{(t, s): 0 \leq s \leq t \leq b\}$

- $A(t)$ and $G(t)$ are closed operators on $X$ with a dense domain $D(A)$ which is independent of $t$;

- $B$ is a bounded linear operator from $V$ to $X$;

- the nonlinear operators $g: J \times \mathcal{B}_{h} \rightarrow X, h: D \times$ $\mathcal{B}_{h} \rightarrow X$ and $f: J \times \mathcal{B}_{h} \times X \rightarrow X$ are continuous;

- $I_{k}: X \rightarrow X, 0=t_{0}<t_{1}<\cdots<t_{k}<t_{k+1}=b$.

Below, we introduce the definitions both of a mild solution and a reachable set of the impulsive neutral functional evolution integro-differential system.

Definition 5. (Radhakrishnan and Balachandran, 2011) A function $x(\cdot) \in \mathcal{P C}((-\infty, b], X)$ is called a mild solution of the dynamic system (3) if

$$
\begin{gathered}
x_{0}=\phi \in \mathcal{B}_{h} \quad \text { on }(-\infty, 0], \\
\left.\Delta x\right|_{t=t_{k}}, \quad k=1,2, \ldots, m ;
\end{gathered}
$$

the restriction of $x(\cdot)$ to the interval $J_{k}(k=0,1, \ldots, m)$ is continuous; for each $t \in[0, b)$, the function $U(t, s) A(s) g\left(s, x_{s}\right), s \in[0, t)$ is integrable and the impulsive integral equation

$$
\begin{aligned}
& x(t) \\
& =U(t, 0)[\phi(0)+g(0, \phi)]-g\left(t, x_{t}\right) \\
& \quad-\int_{0}^{t} U(t, s) A(s) g\left(s, x_{s}\right) \mathrm{d} s \\
& \quad+\int_{0}^{t} U(t, s) \int_{0}^{s} G(s, \tau) x(\tau) \mathrm{d} \tau \mathrm{d} s \\
& \quad+\int_{0}^{t} U(t, s)\left[B u(s)+f\left(s, x_{s}, \int_{0}^{s} h\left(s, \tau, x_{\tau}\right)\right] \mathrm{d} s\right. \\
& \quad+\sum_{0<t_{k}<t} U\left(t, t_{k}\right) I_{k}\left(x\left(t_{k}^{-}\right)\right), \quad t \in J,
\end{aligned}
$$

is satisfied.

Definition 6. (Radhakrishnan and Balachandran, 2011) The reachable set for the system (3) is described by the following formula:

$$
\mathcal{R}\left(b, x_{0}\right)=\left\{x_{b}\left(x_{0} ; u\right)(0): u(\cdot) \in L^{2}(J, V)\right\}
$$

with an initial value $x_{0}=\phi \in \mathcal{B}_{h}$ and a state value $x_{b}\left(x_{0} ; u\right)$ at terminal time $b$ corresponding to control $u$.

Definition 7. (Radhakrishnan and Balachandran, 2011) The system (3) is said to be approximately controllable on the interval $J$ if $\overline{\mathcal{R}\left(b, x_{0}\right)}=X$, where $\overline{\mathcal{R}\left(b, x_{0}\right)}$ is the closure of the set $\mathcal{R}\left(b, x_{0}\right)$ in $X$. 
In order to examine approximate controllability of (3), the following assumptions should be introduced (Radhakrishnan and Balachandran, 2011).

Assumption 5. The strongly continuous semigroup of bounded linear operators $U(t, s)$ generated by $A(t)$ is compact when $t>s>0$ and there exist constants $M_{1}>0, M_{2}>0$ such that

$$
\|U(t, s)\| \leq M_{1}, \quad\|G(t, s)\| \leq M_{2} .
$$

Assumption 6. The function $g: J \times \mathcal{B}_{h} \rightarrow X$ is continuous and there exist constants $L_{g}>0, N_{g}>0$ such that

$$
\left\|g\left(t, \phi_{1}\right)-g\left(s, \phi_{2}\right)\right\| \leq L_{g}\left[|t-s|+\left\|\phi_{1}-\phi_{2}\right\|_{\mathcal{B}_{h}}\right]
$$

for every $t, s \in J$ and $\phi_{1}, \phi_{2} \in \mathcal{B}_{h}$, and

$$
\begin{gathered}
\left\|A(t) g\left(s_{1}, \phi\right)-A(t)\left(s_{2}, \psi\right)\right\| \\
\leq N_{g}\left[\left|s_{1}-s_{2}\right|+\|\phi-\psi\|_{\mathcal{B}_{h}}\right], \\
s_{1}, s_{2} \in J, \quad \phi, \psi \in \mathcal{B}_{h} .
\end{gathered}
$$

Assumption 7. The function $f: J \times B_{h} \times X \rightarrow X$ is continuous and uniformly bounded and there exists $L_{f}>$ 0 such that

$$
\|f(t, \phi, x)\| \leq L_{f} \quad \text { for all }(t, \phi, x) \in J \times \mathcal{B}_{h} \times X .
$$

Theorem 4. (Radhakrishnan and Balachandran, 2011) If Assumptions 1] and 5, 7 are satisfied, then the system (3) is approximately controllable on $J$.

The proof of the above theorem is based on Schauder's fixed-point theorem and presented by Radhakrishnan and Balachandran (2011).

\section{Approximate controllability of stochastic impulsive systems}

In this section, at first we present approximate controllability of stochastic impulsive systems with control-dependent coefficients. Secondly, we describe approximate controllability of nonlinear impulsive differential systems.

4.1. Approximate controllability of stochastic impulsive systems with control-dependent coefficients. The dynamic systems described by the following formula was investigated by Shen and Sun (2011):

$$
\begin{aligned}
\mathrm{d} x(t)= & {[A(t, x(t), u(t)) x(t)} \\
& +B(t, x(t), u(t)) u(t) \\
& +f(t, x(t), u(t))] \mathrm{d} t \\
& +g(t, x(t), u(t)) \mathrm{d} W, \quad t \in J, t \neq \tau_{k}, \quad(5 \mathrm{a}) \\
\Delta & x(t)=I_{k}(x(t)), \quad t=\tau_{k}, \quad k=1,2, \ldots, m,
\end{aligned}
$$

with the initial value $x(0)=x_{0}$. Here $f: J \times \mathbb{R}^{n} \times \mathbb{R}^{n} \rightarrow$ $\mathbb{R}^{n}, g: J \times \mathbb{R}^{n} \times \mathbb{R}^{n} \rightarrow \mathbb{R}^{n \times l}, \Delta x(t)$ denotes the jump of $x$ at $t$, i.e.,

$$
\Delta x(t)=x\left(t^{+}\right)-x\left(t^{-}\right)=x\left(t^{+}\right)-x(t),
$$

and $I_{k} \in C\left(\mathbb{R}^{n}, \mathbb{R}^{n}\right)$. The initial value $x_{0}$ constitutes an $F_{0}$-measurable random variable with $E\left\|x_{0}\right\|^{2}<\infty$. $F_{t}$ is the filtration generated by $w(s), 0 \leq s \leq t$.

Observe that (5) is nonlinear and it is unknown whether such a control exists. Shen and Sun (2011) consider the following dynamic system, instead of the system (5):

$$
\begin{aligned}
\mathrm{d} x(t)= & {[A(t, y(t), v(t)) x(t)} \\
& +B(t, y(t), v(t)) u(t) \\
& +f(t, y(t), v(t))] \mathrm{d} t \\
& +g(t, y(t), v(t)) \mathrm{d} W, \quad t \in[0, T], \quad t \neq \tau_{k},
\end{aligned}
$$

$$
\Delta x(t)=I_{k}(x(t)), \quad t=\tau_{k}, \quad k=1,2, \ldots, m,
$$

where $y, v$ are continuous functions with appropriate dimensions. Now the mild solution of 6 is given by

$$
\begin{aligned}
x(t)= & \Omega(t, y, v) x_{0} \\
& +\int_{0}^{t} \Omega(t-s, y, v)[B(s, y, v) u(s) \\
& +f(s, y, v)] \mathrm{d} s \\
& +\int_{0}^{t} \Phi(t-s, y, v) g(s, y, v) \mathrm{d} W(s) \\
& +\sum_{0<\tau_{k}<t} \Phi\left(t-\tau_{k}, y, v\right) I_{k}\left(x\left(\tau_{k}\right)\right),
\end{aligned}
$$

where $\Phi(t, y, v)$ satisfies

$$
\frac{\mathrm{d} \Phi(t, y, v)}{\mathrm{d} t}=A(t, y, v) \Phi(t, y, v)
$$

with $\Phi(0, y, v)=I$.

To investigate the approximate controllability of (5), the following assumptions are established (Shen and Sun, 2011).

Assumption 8. The linear system (6) is approximately controllable.

Assumption 9. There exist positive constants $M_{1}, d_{k}$, $k=1,2, \ldots, m$, and a function $q(t)$ with a bounded Lebesgue integral such that

$$
\begin{gathered}
\|\Phi(t, y, v)\| \leq M_{1}, \quad\|B(t, y, v)\| \leq q(t), \\
\left\|I_{k}\left(y\left(\tau_{k}\right)\right)\right\| \leq d_{k}, \quad d=\sum_{k=1}^{m} d_{k} .
\end{gathered}
$$


Assumption 10. There holds

$$
\|f(t, x, u)\| \vee\|g(t, x, u)\| \leq \psi(t,\|x\|,\|u\|),
$$

where $\psi^{2}(t, \beta, \gamma)$ is a concave, continuous function in its argument $t$ and nondecreasing for any $\beta>0, \gamma>0$.

Lemma 1. (Mahmudov, 2002) There exists a unique optimal control

$$
\begin{aligned}
& u_{\alpha}(t) \\
= & B^{*} \Phi^{*}(T-t, y, v) \mathbb{E}\left\{\mathcal{R}\left(\alpha, \Pi_{0}^{T}\right) Q(y, v) \mid \mathcal{F}_{t}\right\}
\end{aligned}
$$

such that the functional

$$
J(u)=\mathbb{E}\left\|x\left(T ; x_{0}, u\right)-h\right\|^{2}+\alpha \mathbb{E} \int_{0}^{T}\|u(t)\|^{2} \mathrm{~d} t
$$

takes on its minimum value, where

$$
\begin{aligned}
Q(y, v)= & h-\Phi(T, y, v) x_{0} \\
& -\int_{0}^{T} \Phi(T-s, y, v) f(s, y, v) \mathrm{d} s \\
& -\int_{0}^{T} \Phi(T-s, y, v) g(s, y, v) \mathrm{d} W(s) \\
& -\sum_{k=1}^{m} \Phi\left(T-\tau_{k}, y, v\right) I_{k}\left(x\left(\tau_{k}\right)\right) .
\end{aligned}
$$

The control $u_{\alpha}$ defined by Lemma 1 will provide the solution of (6) approximately to $h$ at $T$ with $h \in \mathcal{L}\left(\Omega, F_{T}, \mathbb{R}^{n}\right)$. Observe that appropriately selected vectors $y, v$ agree with $x, u$. These vectors result from (7), (8), respectively, and are also solutions of the primary problem (5). Then approximate controllability is satisfied. Therefore, approximate controllability of (5) is converted to an existence problem of the fixed point of (7) and (8).

For all $\alpha>0$, the operator $\mathcal{P}_{\alpha}$ on $\mathcal{L}_{2}^{\mathcal{F}_{t}}\left(J \times \Omega, \mathbb{R}^{n}\right) \times$ $\mathcal{L}_{2}^{\mathcal{F}_{t}}\left(J \times \Omega, \mathbb{R}^{n}\right)$ can be defined as

$$
\mathcal{P}_{\alpha}(y, v)=(x, u) .
$$

Theorem 5. (Shen and Sun, 2011) Let Assumptions $8-10$ be satisfied. If there exists a closed bounded convex subset $S$ in $\mathcal{L}_{2}^{\mathcal{F}_{t}}\left(J \times \Omega, \mathbb{R}^{n}\right) \times \mathcal{L}_{2}^{\mathcal{F}_{t}}\left(J \times \Omega, \mathbb{R}^{n}\right)$ such that for all $\alpha>0$ the operator $\mathcal{P}_{\alpha}$ is invariant for $S$, then the system (5) is approximately controllable.

4.2. Approximate controllability of nonlinear impulsive differential systems. In the work of Sakthivel et al. (2007), approximate controllability of the following nonlinear impulsive differential equation is investigated:

$$
\begin{aligned}
\frac{\mathrm{d}}{\mathrm{d} t} x(t) & =A x(t)+(B u)(t)+f(t, x(t)), \\
t & \in J=[0, b] \backslash D, \quad x(0)=x_{0}, \\
\Delta x\left(t_{k}\right) & =I_{k}\left(x\left(t_{k}\right)\right), \quad k=1, \ldots, m,
\end{aligned}
$$

where $A$ is the infinitesimal generator of a strongly continuous semigroup $T(t)$ in a Hilbert space $X, B$ is a linear bounded operator from a Hilbert space $U$ into $X$, $f: J \times X \rightarrow X$ is a nonlinear operator, the control $u(\cdot) \in L_{2}(J, U)$. Here $D=\left\{t_{1}, t_{2}, \ldots, t_{m}\right\} \subset J, 0=$ $t_{0}<t_{1}<\ldots<t_{m}<t_{m+1}=b, \quad I_{k}(k=1,2, \ldots, m)$ is a nonlinear map and $\Delta x\left(t_{k}\right)=x\left(t_{k}^{+}\right)-x\left(t_{k}^{-}\right)=$ $x\left(t_{k}^{+}\right)-x\left(t_{k}\right)$. This represents the jump in the state $x$ at the time $t_{k}$ with $I_{k}$ determining the size of the jump.

Below, we present the definition of approximate controllability of a nonlinear impulsive differential equation.

Definition 8. (Sakthivel et al., 2007) The system (10) is said to be approximately controllable on the interval $J$ if $\overline{\mathcal{R}\left(b, x_{0}\right)}=X$.

Now, we introduce the following assumptions (Sakthivel et al., 2007).

Assumption 11. The semigroup $T(t), t>0$ is compact.

Assumption 12. The function $f: J \times X \rightarrow X$ is continuous and there exist functions $\lambda_{i}(\cdot) \in L_{1}\left(J, \mathbb{R}^{+}\right)$ and $h_{i}(\cdot) \in L_{1}\left(X, \mathbb{R}^{+}\right), i=1,2, \ldots, q$, such that

$$
\|f(t, x)\| \leq \sum_{i=1}^{q} \lambda_{i}(t) h_{i}(x) \quad \text { for all }(t, x) \in J \times X .
$$

Assumption 13. For each $\alpha>0$,

$$
\limsup _{r \rightarrow \infty}\left(r-\sum_{i=1}^{q} \frac{c_{i}}{\alpha} \sup \left\{h_{i}(x):\|x\| \leq r\right\}\right)=\infty,
$$

where

$$
\begin{gathered}
M_{T}=\max \{\|T(t)\|: 0 \leq t \leq b\}, \quad M_{B}=\|B\|, \\
\left\|\lambda_{i}\right\|=\int_{0}^{b}\left|\lambda_{i}(s)\right| \mathrm{d} s, \quad a_{i}=3 k M_{T}^{2} M_{B}\left\|\lambda_{i}\right\|, \\
b_{i}=3 M_{T}\left\|\lambda_{i}\right\|, \quad c_{i}=\max \left\{a_{i}, b_{i}\right\}, \\
k=\max \left\{1, M_{T} M_{B}, M_{T} M_{B} b\right\} .
\end{gathered}
$$

Assumption 14. $I_{k} \in C(X, X)$ and there exists a constant $d_{k}$ such that $\left\|I_{k}(x)\right\| \leq d_{k}$ for each $x \in X, k=$ $1, \ldots, m$.

Assumption 15. The function $f: J \times X \rightarrow X$ is continuous and uniformly bounded and there exists $N>$ 0 such that $\|f(t, x)\| \leq N$ for all $(t, x) \in J \times X$.

It will be shown that the system (10) is approximately controllable if for all $\alpha>0$ there exists a continuous function $x(\cdot) \in C(J, X)$ such that

$$
u(t)=B^{*} T^{*}(b-t) R\left(\alpha, \Gamma_{0}^{b}\right) p(x(\cdot)),
$$




$$
\begin{aligned}
x(t)= & T(t) x_{0} \\
& +\int_{0}^{t} T(t-s)[B u(s)+f(s, x(s))] \mathrm{d} s \\
& +\sum_{0<t_{k}<t} T\left(t-t_{k}\right) I_{k}\left(x\left(t_{k}\right)\right),
\end{aligned}
$$

where

$$
\begin{aligned}
p(x(\cdot))= & x_{b}-T(b) x_{0} \\
& -\int_{0}^{b} T(b-s) f(s, x(s)) \mathrm{d} s \\
& -\sum_{k=1}^{m} T\left(b-t_{k}\right) I_{k}\left(x\left(t_{k}\right)\right) .
\end{aligned}
$$

Theorem 6. (Sakthivel et al., 2007) If Assumptions 11 14 are satisfied, then for all $0<\alpha \leq 1$ the system (10) has a solution on J, and if the Assumptions 11 and $13-15$ hold, then the system (10) is approximately controllable.

\section{Approximate controllability of evolution systems with nonlocal conditions}

Mahmudov (2008) focuses on the following semilinear equation:

$$
\begin{aligned}
x(t)=S & (t) x_{0} \\
& +\int_{0}^{t} S(t-s)[B u(s)+f(x(s))] \mathrm{d} s, \quad t \geq 0 .
\end{aligned}
$$

Let us introduce the following assumptions (Mahmudov, 2008).

Assumption 16. $X$ and $U$ are separable Hilbert spaces, $S(t), t>0$ is a compact semigroup on $X$ and $B \in$ $L(U, X)$.

Assumption 17. $f: X \rightarrow X$ is $C^{1}$ with the Fréchet derivative $f^{\prime}(\cdot)$ such that, for some $L>0$,

$$
\left\|f^{\prime}(z)\right\|_{L(x)} \leq L \quad \text { for all } z \in X .
$$

It is clear that under Assumptions 16 and 17, for any $x_{0} \in X$ and $u(\cdot) \in L^{2}(0, T ; X)$, the system (13) admits a unique solution $x(\cdot)=x\left(\cdot, x_{0}, u\right)$.

Definition 9. (Mahmudov, 2008) Let $x_{0}, h \in X$ and $T>0$. We say that the system (13) is approximately controllable if for any $\varepsilon>0$ there exists $u(\cdot) \in$ $L^{2}(0, T ; X)$ such that

$$
\left\|x\left(T, x_{0}, u\right)-h\right\|<\varepsilon .
$$

Mahmudov (2008) defines an operator given by the following formula:

$$
F(z)=\int_{0}^{1} f^{\prime}(r z) \mathrm{d} r, \quad z \in X
$$

to obtain approximate controllability for the system (13). By Assumption 17 it can be see that there is a constant $L>0$ such that the operator $F$ expressed by (14) has the following properties:

$$
\begin{gathered}
F: X \rightarrow L(X), \quad f(z)=F(z) z+f(0), \\
\|F(z(t))\| \leq L, \quad z(\cdot) \in C(0, T ; X), \quad t \in[0, T], \\
F(\cdot) \in C(X, L(X)) .
\end{gathered}
$$

At this point we present approximate controllability for the system (13). According to Mahmudov (2008), we assume the following.

Assumption 18. The system

$$
\begin{aligned}
x(t)= & S(t) x_{0} \\
& +\int_{0}^{t} S(t-s)[B u(s)+F(s) x(s)] \mathrm{d} s
\end{aligned}
$$

is approximately controllable for any function $F \in$ $L^{\infty}(0, T ; L(X))$.

Theorem 7. (Mahmudov, 2008) Let Assumptions 16 18 hold. Then the system (13) is approximately controllable.

The proof of the above-presented theorem is based on Schauder's fixed point theorem and the lemma below.

Lemma 2. (Mahmudov, 2002) For any $\alpha>0$ the operator $P_{\alpha}(z)$ has a fixed point in $C(0, T ; X)$.

\section{Semilinear functional equations}

In the work of Dauer and Mahmudov (2002), the problem of approximate controllability of dynamic systems given by the following semilinear evolution equation is studied:

$$
\begin{aligned}
& \mathrm{d} x(t)=\left[A x(t)+B u(t)+f\left(t, x_{t}, u(t)\right)\right] \mathrm{d} t, \\
& x_{0}(\theta)=\phi(\theta), \quad \theta \in[-h, 0], \quad t \in(0, T],
\end{aligned}
$$

where

- the state $x(\cdot)$ takes values in a Hilbert space $X$;

- the control $u(\cdot) \in L_{2}([0, T], U)$ takes values in a Hilbert space $U$;

- $C([-h, 0], X)$ is the Banach space of all continuous functions from an interval $[-h, 0]$ to $X$ with the supremum norm; it will be shortly denoted by $C$;

$-\phi \in C$. 
If $x:[-h, T] \rightarrow X$ is a continuous function, then $x_{t}$ is an element in $C$ which has a pointwise definition,

$$
x_{t}(\theta)=x(t+\theta) \quad \text { for } \theta \in[-h, 0] .
$$

The solution of the dynamic system (15) can be described as

$$
\begin{aligned}
x_{t}(0)= & x(t) \\
= & S(t) \phi(0) \\
& +\int_{0}^{t} S(t-s)\left[B u(s)+f\left(s, x_{s}, u(s)\right)\right] \mathrm{d} s,
\end{aligned}
$$$$
x_{0}(\theta)=\phi(\theta), \quad \theta \in[-h, 0], \quad t \in(0, T],
$$

where $S(t)$ is a linear semigroup on $X, B: U \rightarrow X$ is a bounded linear operator.

Definition 10. (Dauer and Mahmudov, 2002) The system given by (16) is approximately controllable on the interval $[0, T]$ if

$$
\overline{\mathcal{R}(T, \phi)}=X
$$

where

$$
\mathcal{R}(T, \phi)=\left\{x_{T}(\phi ; u)(0): u(\cdot) \in L_{2}([0, T], U)\right\}
$$

is a reachable set at time $T$ and $\overline{\mathcal{R}(T, \phi)}$ denotes the closure of $\mathcal{R}(T, \phi)$.

To obtain the results of approximate controllability, the following notation should be introduced:

$$
\begin{gathered}
K=\max \{\|S(t)\|: 0 \leq t \leq T\}, \\
\left\|\lambda_{i}\right\|_{1}=\int_{0}^{T}\left|\lambda_{i}(s)\right| \mathrm{d} s, \quad M=\|B\|, \\
a_{i}=3 k M K^{2}\left\|\lambda_{i}\right\|_{1}, \quad b_{i}=3 K\left\|\lambda_{i}\right\|_{1}, \\
c_{i}=\max \left\{a_{i}, b_{i}\right\} .
\end{gathered}
$$

Now we can establish the following assumptions (Dauer and Mahmudov, 2002).

Assumption 19. The semigroup $S(t), t>0$ is compact.

Assumption 20. The function $f:[0, T] \times C \times U \rightarrow X$ is continuous and there exist functions $\lambda_{i} \in L_{1}\left(I, \mathbb{R}^{+}\right)$and $g_{i} \in L_{1}\left(C \times U, \mathbb{R}^{+}\right), i=1,2, \ldots, q$, such that

$$
\|f(t, \phi, u)\| \leq \sum_{i=1}^{q} \lambda_{i}(t) g_{i}(\phi, u)
$$

for all $(t, \phi, u) \in[0, T] \times C \times U$.

Assumption 21. For each $\alpha>0$,

$\limsup _{r \rightarrow \infty}\left(r-\sum_{i=1}^{q} \frac{c_{i}}{\alpha} \sup \left\{g_{i}(\phi, u):\|(\phi, u)\| \leq r\right\}\right)=\infty$.
Assumption 22. The function $f:[0, T] \times C \times U \rightarrow X$ is continuous and uniformly bounded, i.e., there exists $L>$ 0 such that

$$
\|f(t, \phi, u)\| \leq L
$$

for all $(t, \phi, u) \in[0, T] \times C \times U$.

Assumption 23. Let us introduce two crucial operators:

$$
\begin{aligned}
\Gamma_{0}^{T}= & \int_{0}^{T} S(T-s) B B^{*} S^{*}(T-s) \mathrm{d} s \\
& R\left(\alpha, \Gamma_{0}^{T}\right)=\left(\alpha I+\Gamma_{0}^{T}\right)^{-1} .
\end{aligned}
$$

Furthermore, we assume that $\alpha R\left(\alpha, \Gamma_{0}^{T}\right) \rightarrow 0$ as $\alpha \rightarrow 0^{+}$ in the strong operator topology.

For $\alpha>0$, let us define operator $\mathbb{F}^{\alpha}$ on

$$
C([0, T], C) \times C([0, T], U)
$$

as follows:

$$
\mathbb{F}^{\alpha}(x, u)=(z, \nu)
$$

where

$$
\begin{aligned}
\nu(t)= & B^{*} S^{*}(T-t) R\left(\alpha, \Gamma_{0}^{T}\right) p(x, u), \\
z(t)= & S(t) \phi(\theta) \\
& +\int_{0}^{t} S(t-s)\left(B \nu(s)+f\left(s, x_{s}, u(s)\right)\right) \mathrm{d} s, \\
z_{0}(\theta)= & \phi(\theta), \quad \theta \in[-h, 0], \\
p(x, u)= & x_{T}-S(T) \phi(0) \\
& -\int_{0}^{T} S(T-s) f\left(s, x_{s}, u(s)\right) \mathrm{d} s .
\end{aligned}
$$

Finally, the theorem about approximate controllability of the dynamical system (16) is presented.

Theorem 8. (Dauer and Mahmudov, 2002) Assume that Assumptions 19,22 and 23 are satisfied. Then the system (16) is approximately controllable on $[0, T]$.

Theorem 9. (Dauer and Mahmudov, 2002) Assume that Assumptions 19,20 and 21 are satisfied. Then, for each $0<\alpha \leq 1$, the operator $\mathbb{F}^{\alpha}$ has a fixed point in $C([0, T], C) \times C([0, T], U)$.

Similarly as before, the proof is based on Schauder's fixed point theorem.

\section{Example}

In this section, we focus on the heat control system studied by Hong (1982) and Naito (1987). The heat control system with delays is given by the following formula (Naito, 1987):

$$
\begin{aligned}
\frac{\partial y(t, x)}{\partial t} & =\frac{\partial^{2} y(t, x)}{\partial x^{2}}+y((t-h), x) \\
& +B u(t, x)+f(t, y((t-h), x)), \\
y(t, 0) & =y(t, \pi)=0, \quad t \in[0, T], \\
y(t, x) & =\zeta(t, x), \quad-h \leq t \leq 0,
\end{aligned}
$$


where $\zeta(t, x)$ is continuous, $f:[0, T] \times \mathbb{R} \times \mathbb{R} \rightarrow \mathbb{R}$ is continuous and uniformly bounded. Now, we introduce the operator $A: L^{2}[0, \pi] \rightarrow L^{2}[0, \pi]$ given by

$$
A y=\frac{\mathrm{d}^{2} y}{\mathrm{~d} x^{2}}
$$

and its domain

$$
\begin{aligned}
D(A)= & \left\{y \in L^{2}[0, \pi]: y, \frac{\mathrm{d} y}{\mathrm{~d} x}\right. \text { are absolutely continuous, } \\
& \left.\frac{\mathrm{d}^{2} y}{\mathrm{~d} x^{2}} \in L^{2}[0, \pi], \text { and } y(0)=y(\pi)=0\right\} .
\end{aligned}
$$

Hence

$$
A y=\sum_{n=1}^{\infty}\left(-n^{2}\right)\left(y, \phi_{n}\right) \phi_{n}, \quad y \in D(A),
$$

where

$$
\phi_{n}(x)=\sqrt{\frac{2}{\pi}} \sin n x, \quad n=1,2, \ldots
$$

Note that $\phi_{n}$ is the eigenfunction corresponding to the eigenvalue $\lambda_{n}=-n^{2}$ of the operator $A$.

Obviously, $A$ generates a compact semigroup $S(t), t>0$, in $L^{2}[0, \pi]$. Moreover, the semigroup $S(t)$ is given by

$$
S(t) y=\sum_{n=1}^{\infty} e^{-n^{2} t}\left(y, \phi_{n}\right) \phi_{n}, y \in L^{2}[0, \pi] .
$$

Now, let us define an infinite-dimensional space $U$ denoted by the following formula:

$$
U=\left\{u=\sum_{n=2}^{\infty} u_{n} \phi_{n}(x) \mid \sum_{n=2}^{\infty} u_{n}^{2}<\infty\right\} .
$$

For a given $t \in[0, T]$, the control $u(t, x)$ is taken from the space $U$, defined above with the norm given by

$$
\|u\|_{U}=\left(\sum_{n=2}^{\infty} u_{n}^{2}\right)^{\frac{1}{2}} .
$$

Next, we define a linear continuous mapping $B$ from $U$ to $L^{2}[0, \pi]$ in the form

$$
B u=2 u_{2} \phi_{1}(x)+\sum_{n=2}^{\infty} u_{n} \phi_{n}(x),
$$

and

$$
u=\sum_{n=2}^{\infty} u_{n} \phi_{n} \in U .
$$

Then the linear system given by the following formula:

$$
\begin{gathered}
\dot{y}(t)=A y(t)+B u(t), \\
y(0)=y_{0}
\end{gathered}
$$

corresponding to (17b) is approximately controllable because of the compactness of the semigroup $S(t), t>0$, generated by $A$.

To put it in another way, it can be observed that

$$
B^{*} v=\left(2 v_{1}+v_{2}\right) \phi_{2}(x)+\sum_{n=3}^{\infty} v_{n} \phi_{n}(x)
$$

and

$$
\begin{aligned}
B^{*} S^{*}(t) y= & \left(2 y_{1} e^{-t}+y_{2} e^{-4 t}\right) \phi_{2}(x) \\
& +\sum_{n=3}^{\infty} y_{n} e^{-n^{2} t} \phi_{n}(t)
\end{aligned}
$$

where

$$
v=\sum_{n=1}^{\infty} v_{n} \phi_{n}(x), \quad y=\sum_{n=1}^{\infty} y_{n} \phi_{n}(x) .
$$

Now we get

$$
\left\|B^{*} S^{*}(t) y\right\|=0 \quad \text { for } t \in[0, T] .
$$

Hence we obtain

$$
\begin{aligned}
& \left\|2 y_{1} e^{-t}+y_{2} e^{-4 t}\right\|^{2} \\
& \qquad+\sum_{n=3}^{\infty}\left\|y_{n} e^{-n^{2} t}\right\|^{2}=0 \quad \text { for } t \in[0, T] \\
& \text { nd } \quad y_{n}=0 \quad \text { for } n=1,2, \ldots
\end{aligned}
$$

and

The above-mentioned two equalities imply that $y=0$.

Thus, by Theorem 4.1.7 of Curtain and Zwart (1995, p. 147) concerning approximate controllability of linear systems, we conclude that the linear system (18) corresponding to the system (17b) is approximately controllable and then, by Theorem 8 , the system $17 \mathrm{~b}$ is approximately controllable on interval $[0, T]$, too.

\section{Conclusion}

The presented paper focuses on the approximate controllability problem of different types of dynamical systems. Moreover, it presents results for selected works on the investigated approximate controllability of semilinear dynamical systems. All of the presented research concerning approximate controllability of various kinds of dynamic systems in infinite-dimensional spaces has a common way obtaining the results. Generally speaking, at the beginning we prove that the semilinear system is approximately controllable if the associated linear system is approximately controllable, too. Next, we formulate some assumptions for the semilinear dynamical system. The main role is played by the assumption about the Lipschitz continuity. Then we construct an operator and assume that it is compact. Therefore, we show that 
the operator has a fixed point. At the end, we claim that the existence of a fixed point of the operator implies approximate controllability of the semilinear dynamical system. The proof of the main theorem of all studies is based on the application of Schauder's fixed-point theorem. By making some appropriate assumptions, this can establish approximate controllability results for a wide class of semilinear deterministic and stochastic dynamical systems.

Controllability problems for dynamical systems require the application of various mathematical concepts and methods taken directly from differential geometry, functional analysis, topology and matrix analysis. It should be noticed that there are many unresolved problems for controllability concepts for various types of dynamic systems.

\section{Acknowledgment}

The research presented here was conducted as part of projects funded by the National Science Centre in Poland granted according to the decisions DEC-2014/13/B/ST7/00755, DEC-2012/07/B/ST7/01404 and DEC-2012/07/N/ST7/03236.

\section{References}

Arapostathis, A., George, R.K. and Ghosh, M.K. (2001). On the controllability of a class of nonlinear stochastic systems, Systems \& Control Letters 44(1): 25-34.

Babiarz, A., Czornik, A., Klamka, J. and Niezabitowski, M. (2015a). Controllability of discrete-time linear switched systems with constrains on switching signal, in N.T. Nguyen et al. (Eds.), Intelligent Information and Database Systems, Lecture Notes in Computer Science, Vol. 9011, Springer International Publishing, Berlin, pp. 304-312.

Babiarz, A., Czornik, A., Klamka, J. and Niezabitowski, M. (2015b). The selected problems of controllability of discrete-time switched linear systems with constrained switching rule, Bulletin of the Polish Academy of Sciences: Technical Sciences 63(3): 657-666.

Babiarz, A., Czornik, A. and Niezabitowski, M. (2016). Output controllability of the discrete-time linear switched systems, Nonlinear Analysis: Hybrid Systems 21: 1-10.

Bader, R., Gabor, G. and Kryszewski, W. (1996). On the extension of approximations for set-valued maps and the repulsive fixed points, Bollettino della Unione Matematica Italiana B 10(2): 399-416.

Bader, R. and Kryszewski, W. (1994). Fixed-point index for compositions of set-valued maps with proximally $\infty$-connected values on arbitrary ANR's, Set-Valued Analysis 2(3): 459-480.

Balachandran, K. and Dauer, J. (2002). Controllability of nonlinear systems in Banach spaces: A survey, Journal of Optimization Theory and Applications 115(1): 7-28.
Balachandran, K. and Sakthivel, R. (2001). Controllability of integrodifferential systems in Banach spaces, Applied Mathematics and Computation 118(1): 63-71.

Bashirov, A.E. and Kerimov, K.R. (1997). On controllability conception for stochastic systems, SIAM Journal on Control and Optimization 35(2): 384-398.

Bashirov, A.E. and Mahmudov, N.I. (1999). On concepts of controllability for deterministic and stochastic systems, SIAM Journal on Control and Optimization 37(6): 1808-1821.

Benchohra, M., Gorniewicz, L., Ntouyas, S. and Ouahab, A. (2004). Controllability results for impulsive functional differential inclusions, Reports on Mathematical Physics 54(2): 211-228.

Benchohra, M. and Ouahab, A. (2005). Controllability results for functional semilinear differential inclusions in Fréchet spaces, Nonlinear Analysis: Theory, Methods \& Applications 61(3): 405-423.

Bian, W. (1999). Constrained controllability of some nonlinear systems, Applicable Analysis 72(1-2): 57-73.

Chang, Y.-K. (2007). Controllability of impulsive functional differential systems with infinite delay in Banach spaces, Chaos, Solitons \& Fractals 33(5): 1601-1609.

Curtain, R.F. and Zwart, H. (1995). An Introduction to Infinitedimensional Linear Systems Theory, Springer Science \& Business Media, Berlin.

Czornik, A. and Świerniak, A. (2001). On controllability with respect to the expectation of discrete time jump linear systems, Journal of the Franklin Institute 338(4): 443-453.

Czornik, A. and Świerniak, A. (2004). On direct controllability of discrete time jump linear system, Journal of the Franklin Institute 341(6): 491-503.

Czornik, A. and Świerniak, A. (2005). Controllability of discrete time jump linear systems, Dynamics of Continuous Discrete and Impulsive Systems B: Applications \& Algorithms 12(2): 165-189.

Dauer, J. and Mahmudov, N. (2002). Approximate controllability of semilinear functional equations in Hilbert spaces, Journal of Mathematical Analysis and Applications 273(2): 310-327.

Dauer, J., Mahmudov, N. and Matar, M. (2006). Approximate controllability of backward stochastic evolution equations in Hilbert spaces, Journal of Mathematical Analysis and Applications 323(1): 42-56.

Do, V. (1989). A note on approximate controllability of semilinear systems, Systems \& Control Letters 12(4): 365-371.

Dubov, M. and Mordukhovich, B. (1978). Theory of controllability of linear stochastic systems, Differential Equations 14: 1609-1612.

George, R.K. (1995). Approximate controllability of nonautonomous semilinear systems, Nonlinear Analysis: Theory, Methods \& Applications 24(9): 1377-1393. 
Gorniewicz, L., Granas, A. and Kryszewski, W. (1991). On the homotopy method in the fixed point index theory of multi-valued mappings of compact absolute neighborhood retracts, Journal of Mathematical Analysis and Applications 161(2): 457-473.

Gorniewicz, L., Ntouyas, S. and O'Regan, D. (2005). Controllability of semilinear differential equations and inclusions via semigroup theory in Banach spaces, Reports on Mathematical Physics 56(3): 437-470.

Henríquez, H.R. (2008). Approximate controllability of linear distributed control systems, Applied Mathematics Letters 21(10): 1041-1045.

Hong, X.Z. (1982). A note on approximate controllability for semilinear one-dimensional heat equation, Applied Mathematics and Optimization 8(1): 275-285.

Jeong, J.-M. and Roh, H.-H. (2006). Approximate controllability for semilinear retarded systems, Journal of Mathematical Analysis and Applications 321(2): 961-975.

Klamka, J. (2000). Constrained approximate controllability, IEEE Transactions on Automatic Control 45(9): 1745-1749.

Kryszewski, W. and Zezza, P. (1994). Remarks on the relay controllability of control systems, Journal of Mathematical Analysis and Applications 188(1): 45-65.

Kumlin, P. (2004). A note on fixed point theory, Functional Analysis Lecture, Mathematics, Chalmers \& GU, Gothenburg.

Lasiecka, I. and Triggiani, R. (1991). Exact controllability of semilinear abstract systems with application to waves and plates boundary control problems, Applied Mathematics and Optimization 23(1): 109-154.

Li, M., Wang, M. and Zhang, F. (2006). Controllability of impulsive functional differential systems in Banach spaces, Chaos, Solitons \& Fractals 29(1): 175-181.

Mahmudov, N.I. (2001a). Controllability of linear stochastic systems, IEEE Transactions on Automatic Control 46(5): 724-731.

Mahmudov, N.I. (2001b). Controllability of linear stochastic systems in Hilbert spaces, Journal of Mathematical Analysis and Applications 259(1): 64-82.

Mahmudov, N.I. (2002). On controllability of semilinear stochastic systems in Hilbert spaces, IMA Journal of Mathematical Control and Information 19(4): 363-376.

Mahmudov, N.I. (2003). Approximate controllability of semilinear deterministic and stochastic evolution equations in abstract spaces, SIAM Journal on Control and Optimization 42(5): 1604-1622.

Mahmudov, N.I. (2008). Approximate controllability of evolution systems with nonlocal conditions, Nonlinear Analysis: Theory, Methods \& Applications 68(3): 536-546.
Mahmudov, N. and Zorlu, S. (2003). Controllability of non-linear stochastic systems, International Journal of Control 76(2): 95-104.

Naito, K. (1987). Controllability of semilinear control systems dominated by the linear part, SIAM Journal on Control and Optimization 25(3): 715-722.

Naito, K. (1989). Approximate controllability for trajectories of semilinear control systems, Journal of Optimization Theory and Applications 60(1): 57-65.

Narayanamoorthy, S. and Sowmiya, S. (2015). Approximate controllability result for nonlinear impulsive neutral fuzzy stochastic differential equations with nonlocal conditions, Advances in Difference Equations 2015(1): 1-16.

Pazy, A. (2012). Semigroups of Linear Operators and Applications to Partial Differential Equations, Springer Science \& Business Media, Berlin.

Przeradzki, B. (2012). A generalization of Krasnoselskii fixed point theorem for sums of compact and contractible maps with application, Open Mathematics 10(6): 2012-2018.

Radhakrishnan, B. and Balachandran, K. (2011) Controllability of impulsive neutral functional evolution integrodifferential systems with infinite delay, Nonlinear Analysis: Hybrid Systems 5(4): 655-670.

Sakthivel, R., Mahmudov, N. and Kim, J. (2007). Approximate controllability of nonlinear impulsive differential systems, Reports on Mathematical Physics 60(1): 85-96.

Sakthivel, R., Nieto, J.J. and Mahmudov, N. (2010), Approximate controllability of nonlinear deterministic and stochastic systems with unbounded delay, Taiwanese Journal of Mathematics 14(5): 1777-1797.

Shen, L. and Sun, J. (2011). Approximate controllability of stochastic impulsive systems with control-dependent coefficients, IET Control Theory \& Applications 5(16): 1889-1894.

Sikora, B. and Klamka, J. (2012). On constrained stochastic controllability of dynamical systems with multiple delays in control, Bulletin of the Polish Academy of Sciences: Technical Sciences 60(2): 301-305.

Sirbu, M. and Tessitore, G. (2001). Null controllability of an infinite dimensional SDE with state-and control-dependent noise, Systems \& Control Letters 44(5): 385-394.

Wang, L. (2006). Approximate controllability and approximate null controllability of semilinear systems, Communications on Pure and Applied Analysis 5(4): 953-962.

Zang, Y. and Li, J. (2013). Approximate controllability of fractional impulsive neutral stochastic differential equations with nonlocal conditions, Boundary Value Problems 2013(1): 1-13.

Zhou, H.X. (1983). Approximate controllability for a class of semilinear abstract equations, SIAM Journal on Control and Optimization 21(4): 551-565. 


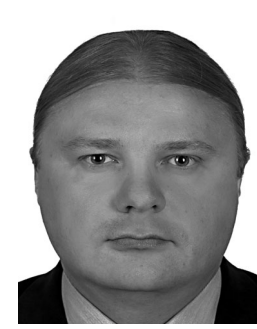

Artur Babiarz received the M.Sc. degree in automatic control from the Silesian University of Technology, Gliwice, Poland, in 2002. From 2002 to 2006, he pursued the Ph.D. degree at the Faculty of Automatic Control there. In 2006, he received the Ph.D. degree in automatic control, with specialization in robotics. Since 2006, he has been an assistant professor and a member of the Control and Robotics Group at the Institute of Automatic Control of the Silesian University of Technology. His research interest includes robot path planning, mathematical modeling of dynamical systems, fundamental study of switched systems, and dynamic properties such as controllability and stability of discrete-time fractional-order and integer-order dynamical systems.

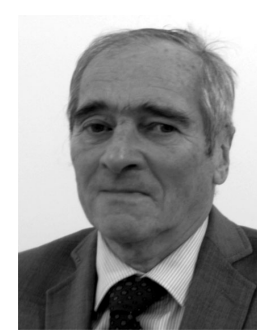

Jerzy Klamka received the $\mathrm{Ph} . \mathrm{D}$. degree in control engineering from the Silesian University of Technology, Gliwice, Poland, in 1974. Moreover, he obtained the Ph.D. degree in mathematics from Silesian University, Katowice, Poland, in 1978. In 1981 he received habilitation in control engineering from the Silesian University of Technology. Since 1990 he has been a full professor of technical sciences there. In 2002 he was elected a corresponding member and in 2010 a full member of the Polish Academy of Sciences. His main area of research includes controllability, observability and stability of linear and nonlinear dynamical systems, and mathematical foundations of quantum computations. He is the author of monographs and more than 300 papers published in international journals.

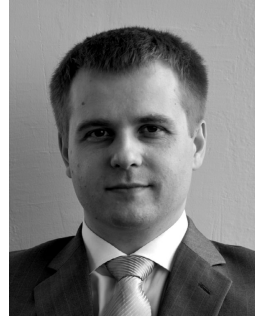

Michał Niezabitowski was born in 1985. In 2005, he started an M.Sc. programme in automatic control and robotics at the Silesian University of Technology, in Poland. After graduation in 2010, he commenced research in control theory at the same university, received a Ph.D. in 2014 and was employed as a research assistant. Now he is a member of the Control and Robotics Group at the Institute of Automatics. His research interests focus on numerical characteristics, controllability and stability of discrete linear systems.

Received: 14 September 2015

Revised: 16 December 2015

Accepted: 29 January 2016 